\title{
Treatment of Breast Cancer Brain Metastases
}

\author{
Rachel A. Freedman and \\ Dana-Farber Cancer Institute, 450 Brookline Avenue, Boston, MA 02215, USA, \\ rafreedman@partners.org
}

Carey K. Anders

University of North Carolina Lineberger Comprehensive Cancer Center, 170 Manning Drive, CB \#7305, Chapel Hill, NC 27599-7305, USA, carey_anders@med.unc.edu

\begin{abstract}
Approximately $10 \%$ to $15 \%$ of women with metastatic breast cancer will develop brain metastases. Treatment options for these women remain limited, particularly at the time of central nervous system (CNS) relapse following completion of initial CNS-directed therapy. Historically, prior studies have broadly examined systemic treatments for breast cancer brain metastases with mixed, but overall disappointing, results. More recently, studies have increasingly selected patients based on breast cancer subtype and have examined novel, targeted agents that have preclinical suggestion of blood-brain barrier penetration. Correlative science objectives, with both tissue-based and novel imaging endpoints, are more frequently incorporated into trials of this nature, with the goal of enhancing our understanding of possible predictors of response. This review summarizes the current and emerging data on systemic therapy for breast cancer brain metastases and provides a framework for future directions in treating this clinically-challenging entity.
\end{abstract}

\section{Keywords}

Brain; Metastases; Breast cancer; Systemic therapy; Radiation; Targeted therapy

\section{Introduction}

Brain metastases from breast cancer are the second most common cause of central nervous system (CNS) metastases [1], and approximately $10 \%$ to $15 \%$ of patients with metastatic breast cancer will eventually develop CNS disease [1-4]. Patients with triple negative or human epidermal growth factor receptor 2 (HER2)-positive cancers [5-7] have a particularly high rate of CNS metastases; other risk factors for development of CNS disease include young age, African American ancestry, visceral involvement, and high-grade disease [1,8].

CNS disease may become a sanctuary site for some women with HER2-positive metastatic breast cancer [9]. This has become particularly evident in women who experience prolonged control of systemic disease as a result of improvements in agents that do not cross the blood-brain barrier (BBB), such as trastuzumab. As indirect evidence for this, whereas older case series have reported median survival times of less than 6 months once women with

(C) Springer Science+Business Media, LLC 2011

Correspondence to: Carey K. Anders.

Disclosure R.A. Freedman: none; C.K. Anders has received grants from BCRF-AACR and CALGB, travel expenses covered by BiPAR/ Sanofi-Aventis, and royalties from Jones and Bartlett publishers for the book 100 Questions and Answers About Triple Negative Breast Cancer. 
HER2-positive disease experience CNS recurrence [8, 9], more recent analyses have observed median survival times approaching 2 years $[10,11]$. In addition, a recent update from the joint analysis of the North Central Cancer Treatment Group (NCCTG) N9831 Intergroup trial and the National Surgical Adjuvant Breast and Bowel Project (NSABP) B-31 trial reported a numerically higher rate of CNS relapse for women who received adjuvant trastuzumab despite a lower overall disease event rate in the trastuzumabcontaining arms. However, rates of CNS events were low in both N9831 and B-31 (1.6\%$2.0 \%$ in chemotherapy alone arms vs $3.0 \%-3.1 \%$ in trastuzumab-containing arms) [12].

Women with metastatic triple negative breast cancer may have even higher rates of CNS involvement. In a case review of 116 women at Dana-Farber Cancer Institute (Boston, MA) with triple negative metastatic disease, $14 \%$ of women developed CNS disease as their initial site of recurrence and $46 \%$ of women developed CNS metastases before death [13]. Unfortunately, median survival times remain short in this subset of women, with most series reporting less than 6 months from onset of CNS disease until death [10, 13•, 14-16].

Although the standard, up-front approach for treatment of CNS disease across all breast cancer subtypes typically includes surgery, radiation, or a combination of local therapy modalities, systemic therapy has become an increasingly appealing option because of the potential ability to avoid or delay whole brain radiation therapy (WBRT) and its associated complications while concurrently treating systemic disease. Moreover, studies have illustrated that the addition of systemic therapy, both chemotherapy and targeted agents, following WBRT translates into improved survival, particularly among patients with endocrine sensitive and HER2-positive breast cancer brain metastases [17]. However, as no systemic therapy has been approved by the US Food and Drug Administration (FDA) for the treatment of breast cancer brain metastases, this clinical entity presents a significant treatment challenge for providers, particularly when CNS relapses occur. This review summarizes the emerging data on systemic therapy of breast cancer brain metastases.

\section{Overview of Local Therapies to Treat Breast Cancer Brain Metastases}

Although systemic therapy certainly plays an integral role in the treatment paradigm for patients with breast cancer brain metastases, local therapy, including surgery, radiosurgery, and WBRT, is paramount in the initial and subsequent management of metastatic brain tumors [18]. There are many variables to consider as one embarks on an initial treatment plan, including prognostic factors, patient preference, status of systemic/extracranial disease, number, size and/or location of metastases, time from initial diagnosis to development of brain metastases, and expertise available at the treating center. In general, patients with solitary brain metastases can be considered for upfront surgical resection or radiosurgery depending on resectability of the lesion, often followed by WBRT (http://www.nccn.org). This approach has translated into improved survival for patients with solitary brain metastases compared to WBRT alone across several solid tumor types [19, 20]. More recently, studies have explored the efficacy and safety of neurosurgery and/or radiosurgery followed by observation or WBRT among patients with one to three brain metastases in effort to avoid the long-term consequences, namely neurocognitive decline, of WBRT. In general, these studies have illustrated higher rates of intracranial recurrence/relapse with similar to improved neurocognition/functional independence for those treated with observation versus WBRT following upfront neurosurgical resection or radiosurgery [21-23]. Overall survival has been similar between both groups. For patients with multiple brain metastases or uncontrolled extracranial disease, WBRT as a single modality remains the recommended treatment strategy. The complexity of upfront management of metastatic breast cancer to the CNS requires a multi-disciplinary approach, including input from multiple subspecialties such as neuroradiology, neurosurgery, radiation oncology, and medical oncology. 


\section{Chemotherapy for Treatment of Breast Cancer Brain Metastases}

Until recently, clinical trials for patients with CNS recurrence have typically included administration of standard chemotherapy agents, as single agents or in combination, to patients with a variety of solid tumors (including breast cancer) or to women with breast cancer in a non-subtype-specific manner. Past treatment trials for women with breast cancer brain metastases have examined efficacy of treatments such as temozolomide [24-27], capecitabine-temozolomide [28], topotecan [29], liposomal doxorubicin-temozolomide [30], cisplatin-etoposide [31], and cisplatin-temozolomide [32]. Response rates in these studies have ranged from 0\% to 38\%, with progression-free survival (PFS) times of 0 to 6 months (Table 1). One additional study in Singapore attempted to administer liposomal doxorubicin to patients with breast cancer and recurrent CNS disease but terminated prematurely due to slow accrual. Case series and case reports in breast cancer brain metastases have also described mixed results with combination therapies such as cyclophosphamide-methotrexate-5-fluorouracil [33], cyclophosphamide-doxorubicin-5fluorouracil [33], cyclophosphamide-5-fluorouracil-prednisone [34], doxorubicincyclophosphamide [34], paclitaxel [35], and carmustine-methotrexate [36]. However, the small sample sizes and tumor heterogeneity included in these studies have limited their impact and applicability in clinical practice.

A notable combination therapy specifically for patients with breast cancer brain metastases is that of temozolomide-irinotecan. A phase II study enrolled 17 patients with progressive brain metastases or leptomeningeal disease after receipt of WBRT who were treated with irinotecan $125 \mathrm{mg} / \mathrm{m}^{2}$ every 14 days and temozolomide $100 \mathrm{mg} / \mathrm{m}^{2}$ on days 1 to 7 and 15 to 21 [37]. Median time to progression in the CNS was 11.5 weeks (range, 4-63 weeks) for all patients; 16 weeks (range, 8-63 weeks) for those with brain metastases only, and 4.5 weeks (range, 4-10 weeks) for those with leptomeningeal disease. One patient had a partial response in the brain at week 46, and a second patient maintained stable disease for 56 weeks. Adverse events were mild and included grade 1 to 2 neutropenia, diarrhea, nausea, vomiting, and fatigue. Updated results from this intriguing study are anticipated.

Recent studies for this patient population have increasingly incorporated novel chemotherapeutic agents in addition to embedded correlative science endpoints in an effort to ascertain potential predictors of response. Epothilones are a new class of tubulinstabilizing agents that have demonstrated responses in pretreated, metastatic breast cancer [38-40]. Sagopilone is an epothilone that is not a substrate of P-glycoprotein and readily crosses the BBB in preclinical studies [41, 42], with preliminary activity initially reported in gliomas [43, 44]. In a single center phase II study, sagopilone was administered at $16 \mathrm{mg} / \mathrm{m}^{2}$ or $22 \mathrm{mg} / \mathrm{m}^{2}$ every 21 days to patients with progressive brain metastases from breast cancer of any subtype [45]. Although two patients achieved a partial response (13.3\%), the trial prematurely closed to accrual after 15 patients enrolled because of short PFS duration (median of 1.4 months) in addition to the evolving, somewhat disappointing data with regard to sagopilone monotherapy for treatment of systemic disease [46]. As previous work suggested that CNS vessel tortuosity observed with malignancy may normalize when cancers respond to treatment $[47,48]$, this trial also incorporated correlative imaging studies with high-resolution magnetic resonance angiography (MRA). Although the small sample size did not allow for conclusions to be drawn regarding these correlative analyses, similar correlative analyses in a larger patient cohort have been incorporated into an ongoing study with carboplatin and bevacizumab at the Dana-Farber Cancer Institute (NCT01004172).

Patupilone, a natural epothilone $\mathrm{B}$, has shown more promising results for treatment of CNS disease. In a preliminary report of 38 patients with progressive breast cancer brain metastases who received patupilone $10 \mathrm{mg} / \mathrm{m}^{2}$ every 21 days, $13 \%$ of women $(n=5)$ experienced a partial response in the CNS and 5 additional patients experienced stable 
disease. The three-month PFS in the CNS was 37\% [49], reaching the threshold to proceed with further study of this agent for this population.

In addition to the epothilones, ANG1005 (now known as GRN1005, GERON) is a novel angiopep-2-paclitaxel conjugate formulated to cross the BBB by binding the LRP-1 receptor (low-density lipoprotein receptor-related protein) located on the BBB. In preclinical models, administration of ANG1005 resulted in higher intracranial tumor paclitaxel content when compared to that of standard paclitaxel administration [50]. A phase I trial of ANG1005 has also recently completed accrual for patients with solid tumors (25\% [14/56], breast cancer), with and without brain metastases. A maximum-tolerated dose was established and preliminary efficacy (both intra- and extracranially) and safety results were promising [51]. In addition, a phase I trial of ANG1005 in patients with malignant gliomas suggested activity [52], and future study in the setting of solid tumor brain metastases is anticipated.

\section{HER2-Directed Therapy}

Few prospective studies have examined administration of systemic, targeted therapy to treat women with HER2-positive breast cancer brain metastases. The largest study reported to date was a multicenter study in which lapatinib, an oral epidermal growth factor receptor (EGFR)/HER2-inhibitor, was administered to women with progressive CNS disease [53•]. Of the 242 patients enrolled in the study, $6 \%$ experienced an objective response rate (ORR) in the CNS (95\% CI, 3.6-10.2), defined by presence of the following composite criteria: 1) $\geq 50 \%$ volumetric reduction of CNS lesion(s), 2) absence of increasing steroid use, 3 ) absence of progressive neurologic symptoms, and 4) absence of progressive extracranial disease. Of the 50 patients who were offered extension phase therapy with lapatinib and cape-citabine at progression, 20\% experienced an ORR and 40\% experienced at least a $20 \%$ volumetric reduction in CNS disease. Although the observed ORR for lapatinib monotherapy was modest, this study demonstrated that administration of lapatinib with chemotherapy partners such as capecitabine may enhance efficacy. In addition, this trial demonstrated that large, prospective studies in this patient population are feasible.

A second study has demonstrated the efficacy of lapatinib and capecitabine in the setting of progressive CNS disease. In a phase II study of lapatinib in combination with topotecan or capecitabine for women with progressive, HER2-positive breast cancer brain metastases, 22 of a planned 110 patients were enrolled before study closure due to excessive toxicity and lack of efficacy in the lapatinib and topotecan arm (0 responses). However, in the capecitabine and lapatinib arm, 38\% of women experienced a partial response (PR) (95\% CI, 13.9-68.4) [54].

These data have been further reinforced by recently presented data from the LANDSCAPE trial [55•]. In this study, 45 women with radiation therapy-naïve, clinically stable, HER2positive breast cancer brain metastases received lapatinib at $1,250 \mathrm{mg} /$ day and capecitabine at $2,000 \mathrm{mg} / \mathrm{m}^{2} / \mathrm{day}$, on days 1 to 14 of a 21 -day cycle. The CNS ORR was $67 \%$ (95\% CI, $51 \%-81 \%$ ), using Lin et al.'s [53•] criteria for response, median time to WBRT was 8.3 months (95\% CI, 5.1-11.7 months), and median time to progression was 5.5 months (95\% CI, 3.9-5.9 months). The most common side effects were diarrhea and hand-foot syndrome in $20 \%$ of patients. Correlative analyses of the predictive abilities of circulating tumor cells were also included [55]. Although further comparison of first-line CNS-directed therapy certainly is warranted, these results provide a new treatment option for a select group of women with newly diagnosed HER2-positive breast cancer brain metastases who are clinically stable and wish to delay upfront WBRT.

Additional studies have evaluated HER-2 directed therapy as a radiosensitizer, combining local and systemic therapy as a way to enhance brain metastases response. A phase I study 
investigated the combination of lapatinib in combination with WBRT for patients with at least one new or progressive HER2-positive brain metastasis. Thirty-five patients received $750 \mathrm{mg}$ of lapatinib orally twice daily on day 1 , followed by $1,000 \mathrm{mg}, 1,250 \mathrm{mg}$, or 1,500 mg once daily. WBRT ( 37.5 Gy in 15 fractions) was started 1 to 8 days after lapatinib initiation, and lapatinib was continued throughout WBRT. After completion of WBRT, patients received trastuzumab $2 \mathrm{mg} / \mathrm{kg}$ weekly and lapatinib 1,000 mg once daily. At 2 months, the ORR in the CNS was 70\% (95\% CI, 50\%-86\%) by predefined volumetric criteria. Despite this observed efficacy, the trial did not meet the predefined criteria for feasibility because 7 of 27 patients experienced dose-limiting toxicities, most commonly rash and diarrhea [56]. Although this treatment combination is still of great interest when considering future trial design, rigorous safety monitoring and a more tolerable dosing schedule would be required.

A second phase I study was recently completed at Jules Bordet in Belgium, in which temozolomide and lapatinib were administered at varying doses to patients with HER2positive breast cancer brain metastases that either recurred after local therapy or were previously untreated. The primary endpoint of this study was to determine the maximum tolerated dose of this treatment combination. After enrollment of 17 patients, a dose-limiting toxicity was observed at the combination of lapatinib $1,500 \mathrm{mg} /$ day and temozolomide 200 $\mathrm{mg} / \mathrm{m}^{2}$ (days 1-5), with an ongoing extension to six patients at this dose level. On preliminary efficacy analyses, 4 of 13 evaluable patients experienced volumetric reduction of their largest CNS lesion, with a median PFS of 2.79 months (95\% CI, 1.8-3.6) and an OS of 10.9 months for all 17 women. Final results from this study are forthcoming [57].

A summary of the available data for protocols targeting HER2-positive disease is provided in Table 2.

\section{Ongoing and Planned HER2-Directed Studies}

Multiple studies are planned and/or ongoing for women with HER2-positive breast cancer brain metastases. This includes a single institution study at Dana-Farber Cancer Institute that is ongoing and will enroll 40 patients with progressive HER2-positive or HER2-negative CNS disease. In this study, patients will receive carboplatin and bevacizumab on an every 21-day cycle with concurrent trastuzumab if HER2-positive. Correlative studies include an examination of CNS vessel tortuosity and response using MRA, the association of survival and circulating tumor cell count, and the possible associations of vascular endothelial growth factor receptor genotype with toxicity and response (NCT01004172).

A second multicenter trial being led by investigators at the University of North Carolina will evaluate the efficacy and safety of the mTOR (mammalian target of rapamycin) inhibitor RAD001 (everolimus, Novartis) in combination with vinorelbine and trastuzumab to treat patients with progressive HER2-positive breast cancer brain metastases (NCT01305941). All patients will have received prior WBRT and/or radiosurgery. Interestingly, RAD001 has been approved by the FDA to treat patients with intracranial subependymal giant cell astrocytoma (SEGA) tumors associated with tuberous sclerosis who are not candidates for neurosurgical resection.

Finally, a multicenter, phase II study of neratinib, an oral irreversible-binding inhibitor of the erbB family of receptor tyrosine kinases that inhibits signal transduction through erbB1 (EGFR), HER2, and erbB4, will soon open through the Translational Breast Cancer Research Consortium. This trial will enroll 40 women with progressive, HER2-positive breast cancer brain metastases in addition to 5 patients who are surgical candidates for craniotomy (with no history of previous CNS-directed therapy). These five patients will receive neratinib preoperatively and will have intracranial specimens and cerebrospinal fluid 
examined at surgery to determine drug concentrations in relation to plasma. This novel approach will provide direct in vivo data on penetration of neratinib into the CNS.

Neurocognitive testing will be administered to all patients on study and circulating tumor cell studies have also been incorporated to examine potential predictors of survival and response.

Other related studies have focused on the development of CNS disease as an endpoint among women with HER2-positive breast cancer who are known to be at higher risk for brain metastases. One such study is currently randomizing women with HER2-positive, metastatic breast cancer without CNS involvement to receive lapatinib plus capecitabine or trastuzumab plus capecitabine (NCT00820222). This study aims to enroll 650 women from multiple centers worldwide with the primary endpoint of CNS disease as the first site of relapse. The Adjuvant Lapatinib And/Or Trastuzumab Treatment Optimization (ALTTO) study has also included development of brain metastases as a first site of recurrence as a secondary endpoint, with results forthcoming.

\section{Antiangiogenic Therapy}

As both primary brain tumors and brain metastases are highly vascular lesions, antiangiogenic agents present an attractive therapeutic strategy. Interestingly, all $(n=22)$ patients with progressive HER2-positive breast cancer brain metastases included in a study of lapatinib monotherapy were found to have abnormal vessel tortuosity at baseline via brain MRA [58]. At present, bevacizumab (Avastin, Genentech/Roche), a monoclonal antibody that targets vascular endothelial growth factor (VEGF), is FDA-approved to treat aggressive primary brain tumors, namely glioblastoma multiforme (GBM). The activity of bevacizumab in primary brain tumors [59] and in metastatic breast cancer [60] has prompted an ongoing phase II study evaluating carboplatin plus bevacizumab (plus trastuzumab if HER2-positive) in the setting of progressive breast cancer brain metastases (NCT01004172), as described above. Although retrospective studies have reported low rates of intracranial hemorrhage among patients with solid tumors (including breast cancer) and brain metastases treated with bevacizumab (1\%-3\%), the results of this study, including both safety and efficacy, are eagerly awaited [61]. Although the concept of antiangiogenic therapy as a radiosensitizer has been explored, a study evaluating the small molecule inhibitor of VEGF, cedirinb (AZD2171), in combination with WBRT closed after only three patients enrolled due to slow accrual (NCT00937482).

\section{Other Novel Agents and Ongoing Trials}

Given the potential to improve outcome for women with breast cancer brain metastases through careful selection of systemic therapy in concert with established local therapies, brain permeability of many of the emerging and novel therapies pertinent to the treatment of breast cancer is of great interest, both in the preclinical and clinical arenas. Such an example includes the PARP (poly-ADP-ribose polymerase) inhibitor ABT-888 (veliparib, Abbott) which has been shown in preclinical studies to penetrate the BBB in an intracranial glioma model [62]. Presently, a phase I study is evaluating the maximum tolerated dose of ABT-888 in combination with conventional WBRT to treat patients with solid tumors metastatic to the brain (NCT00649207). In addition, the efficacy and tolerability of the novel anticancer agent BSI-201 (iniparib, BiPAR Sciences/Sanofi-Aventis) in combination with irinotecan is being examined in a phase II, multicenter study enrolling patients with progressive triple negative breast cancer brain metastases (NCT01173497) [63]. Finally, a recent report illustrates a high rate of Phosphatidylinositol 3-Kinase (PI3K) pathway activation among a panel of breast cancer brain metastases tissues [64]. Given that there are many PI3K inhibitors in clinical development, some of which cross the BBB, targeting this pathway to treat patients with breast cancer brain metastases is also of great interest. 


\section{Conclusions}

Treatment of brain metastases from breast cancer remains a significant clinical challenge, particularly due to the diversity of patients who develop recurrent disease across the breast cancer subtypes, the variation in the status of extracranial disease, receipt of and sensitivity to prior anticancer agents, and the ranges in overall survival. As our ability to control systemic disease continues to improve, CNS recurrence from breast cancer will likely be an increasingly common clinical challenge, particularly for patients with advanced HER2positive and triple negative breast cancer.

Prior experiences illustrate that that multicenter studies, particularly targeting a specific breast cancer subtype, are feasible. This approach allows for enhanced accrual, incorporation of multiple scientific questions and collaboration, and the potential for prospective, randomized studies that have been lacking in this patient population. Although there are multiple opportunities for administration of systemic therapy to treat patients with breast cancer brain metastases, the optimal timing remains uncertain. As evidenced by the aforementioned data, multiple approaches have been investigated including administration of systemic therapy 1) after standard (local) therapy, 2) as up-front therapy in select cases, and 3) as radiation sensitizers. To ascertain the ideal approach, we should strive to select our study populations carefully and to incorporate relevant correlative science objectives into our clinical trial designs such that we can most optimally achieve durable responses, understand predilection to the CNS, and possible predictors of response. Additional areas for ongoing and future study include investigating ways to enhance intracranial radiographic response assessment, improving prediction of patients at highest risk for brain metastases, and developing possible prevention strategies. The ability to adequately address each of these challenges to improve outcomes for women with breast cancer brain metastases will most certainly require a united approach and multidisciplinary team with expertise in local and systemic therapies, as well as basic, translational, and clinical investigation.

\section{References}

Papers of Particular Interest, Published Recently, Have Been Highlighted As:

\section{- $\quad$ Of Importance}

1. Barnholtz-Sloan JS, Sloan AE, Davis FG, et al. Incidence proportions of brain metastases in patients diagnosed (1973 to 2001) in the Metropolitan Detroit Cancer Surveillance System. J Clin Oncol. 2004; 22:2865-72. [PubMed: 15254054]

2. Tsukada Y, Fouad A, Pickren JW, Lane WW. Central nervous system metastasis from breast carcinoma. Autopsy study Cancer. 1983; 52:2349-54.

3. Patanaphan V, Salazar OM, Risco R. Breast cancer: metastatic patterns and their prognosis. South Med J. 1988; 81:1109-12. [PubMed: 3420442]

4. Engel J, Eckel R, Aydemir U, et al. Determinants and prognoses of locoregional and distant progression in breast cancer. Int J Radiat Oncol Biol Phys. 2003; 55:1186-95. [PubMed: 12654426]

5. Bendell JC, Domchek SM, Burstein HJ, et al. Central nervous system metastases in women who receive trastuzumab-based therapy for metastatic breast carcinoma. Cancer. 2003; 97:2972-7. [PubMed: 12784331]

6. Clayton AJ, Danson S, Jolly S, et al. Incidence of cerebral metastases in patients treated with trastuzumab for metastatic breast cancer. Br J Cancer. 2004; 91:639-43. [PubMed: 15266327]

7. Stemmler HJ, Kahlert S, Siekiera W, et al. Characteristics of patients with brain metastases receiving trastuzumab for HER2 overexpressing metastatic breast cancer. Breast. 2006; 15:219-25. [PubMed: 16026983]

8. Tham YL, Sexton K, Kramer R, et al. Primary breast cancer phenotypes associated with propensity for central nervous system metastases. Cancer. 2006; 107:696-704. [PubMed: 16826579] 
9. Yau T, Swanton C, Chua S, et al. Incidence, pattern and timing of brain metastases among patients with advanced breast cancer treated with trastuzumab. Acta Oncol. 2006; 45:196-201. [PubMed: 16546866]

10. Eichler AF, Kuter I, Ryan P, et al. Survival in patients with brain metastases from breast cancer: the importance of HER-2 status. Cancer. 2008; 112:2359-67. [PubMed: 18361426]

11. Melisko ME, Moore DH, Sneed PK, et al. Brain metastases in breast cancer: clinical and pathologic characteristics associated with improvements in survival. J Neurooncol. 2008; 88:35965. [PubMed: 18398574]

12. Perez EA, Romond EH, Suman VJ, et al. Four-year follow-up of trastuzumab plus adjuvant chemotherapy for operable human epidermal growth factor receptor 2-positive breast cancer: joint analysis of data from NCCTG N9831 and NSABP B-31. J Clin Oncol. 2011; 29:3366-73. [PubMed: 21768458]

13. Lin NU, Claus E, Sohl J, et al. Sites of distant recurrence and clinical outcomes in patients with metastatic triple-negative breast cancer: high incidence of central nervous system metastases. Cancer. 2008; 113:2638-2645. This influential article defines the rate of brain metastases among women with advanced triple negative breast cancer at 46\%. [PubMed: 18833576]

14. Dawood S, Broglio K, Esteva FJ, et al. Survival among women with triple receptor-negative breast cancer and brain metastases. Ann Oncol. 2009; 20:621-7. [PubMed: 19150943]

15. Nam BH, Kim SY, Han HS, et al. Breast cancer subtypes and survival in patients with brain metastases. Breast Cancer Res. 2008; 10:R20. [PubMed: 18307763]

16. Hines SL, Vallow LA, Tan WW, et al. Clinical outcomes after a diagnosis of brain metastases in patients with estrogen- and/or human epidermal growth factor receptor 2-positive versus triplenegative breast cancer. Ann Oncol. 2008; 19:1561-5. [PubMed: 18534964]

17. Niwińska A, Murawska M, Pogoda K. Breast cancer subtypes and response to systemic treatment after whole-brain radiotherapy in patients with brain metastases. Cancer. 2010; 116:4238-47. [PubMed: 20549816]

18. Ewend MG, Morris DE, Carey LA, et al. Guidelines for the Initial Management of Metastatic Brain Tumors: Role of Surgery. Radiosurgery, and Radiation Therapy. J Natl Compr Canc Netw. 2008; 6:505-14. [PubMed: 18492462]

19. Patchell RA, Tibbs PA, Walsh JW, et al. A randomized trial of surgery in the treatment of single metastases to the brain. N Engl J Med. 1990; 322:494-500. [PubMed: 2405271]

20. Andrews DW, Scott CB, Sperduto PW, et al. Whole brain radiation therapy with or without stereotactic radiosurgery boost for patients with one to three brain metastases: phase III results of the RTOG 9508 randomised trial. Lancet. 2004; 363:1665-72. [PubMed: 15158627]

21. Aoyama H, Tago M, Kato N, et al. Neurocognitive function of patients with brain metastasis who received either whole brain radiotherapy plus stereotactic radiosurgery or radiosurgery alone. Int $\mathbf{J}$ Radiat Oncol Biol Phys. 2007; 68:1388-95. [PubMed: 17674975]

22. Kocher M, Soffietti R, Abacioglu U, et al. Adjuvant Whole-Brain Radiotherapy Versus Observation After Radiosurgery or Surgical Resection of One to Three Cerebral Metastases:

Results of the EORTC 22952-26001 Study. J Clin Oncol. 2011; 29:134-41. [PubMed: 21041710]

23. Chang EL, Wefel JS, Hess KR, et al. Neurocognition in patients with brain metastases treated with radiosurgery or radiosurgery plus whole-brain irradiation: a randomised controlled trial. Lancet Oncol. 2009; 10:1037-44. [PubMed: 19801201]

24. Trudeau ME, Crump M, Charpentier D, et al. Temozolomide in metastatic breast cancer (MBC): a phase II trial of the National Cancer Institute of Canada-Clinical Trials Group (NCIC-CTG). Ann Oncol. 2006; 17:952-6. [PubMed: 16565212]

25. Abrey LE, Christodoulou C. Temozolomide for treating brain metastases. Semin Oncol. 2001; 28:34-42. [PubMed: 11550137]

26. Christodoulou C, Bafaloukos D, Kosmidis P, et al. Phase II study of temozolomide in heavily pretreated cancer patients with brain metastases. Ann Oncol. 2001; 12:249-54. [PubMed: 11300333]

27. Siena S, Crino L, Danova M, et al. Dose-dense temozolomide regimen for the treatment of brain metastases from melanoma, breast cancer, or lung cancer not amenable to surgery or radiosurgery: a multicenter phase II study. Ann Oncol. 2010; 21:655-61. [PubMed: 19767314] 
28. Rivera E, Meyers C, Groves M, et al. Phase I study of capecitabine in combination with temozolomide in the treatment of patients with brain metastases from breast carcinoma. Cancer. 2006; 107:1348-54. [PubMed: 16909414]

29. Oberhoff C, Kieback DG, Wurstlein R, et al. Topotecan chemotherapy in patients with breast cancer and brain metastases: results of a pilot study. Onkologie. 2001; 24:256-60. [PubMed: 11455218]

30. Caraglia M, Addeo R, Costanzo R, et al. Phase II study of temozolomide plus pegylated liposomal doxorubicin in the treatment of brain metastases from solid tumours. Cancer Chemother Pharmacol. 2006; 57:34-9. [PubMed: 16010592]

31. Franciosi V, Cocconi G, Michiara M, et al. Front-line chemotherapy with cisplatin and etoposide for patients with brain metastases from breast carcinoma, nonsmall cell lung carcinoma, or malignant melanoma: a prospective study. Cancer. 1999; 85:1599-605. [PubMed: 10193952]

32. Christodoulou C, Bafaloukos D, Linardou H, et al. Temozolomide (TMZ) combined with cisplatin (CDDP) in patients with brain metastases from solid tumors: a Hellenic Cooperative Oncology Group (HeCOG) Phase II study. J Neurooncol. 2005; 71:61-5. [PubMed: 15719277]

33. Boogerd W, Dalesio O, Bais EM, van der Sande JJ. Response of brain metastases from breast cancer to systemic chemotherapy. Cancer. 1992; 69:972-80. [PubMed: 1735089]

34. Rosner D, Nemoto T, Lane WW. Chemotherapy induces regression of brain metastases in breast carcinoma. Cancer. 1986; 58:832-9. [PubMed: 3755076]

35. Kokufu I, Tanei T, Taniguchi H, et al. A case of effective paclitaxel therapy for adriamycin resistant metastatic breast cancer with brain metastases. Gan To Kagaku Ryoho. 2002; 29:585-8. [PubMed: 11977543]

36. Jacot W, Gerlotto-Borne MC, Thezenas S, et al. Carmustine and methotrexate in combination after whole brain radiation therapy in breast cancer patients presenting with brain metastases: a retrospective study. BMC Cancer. 2010; 10:257. [PubMed: 20525352]

37. Melisko, ME.; Anderson, M.; Scott, J., et al. Phase II study of irinotecan (IN) and temozolomide (TMZ) in breast cancer patients (pts) with brain metastases (BM) or leptomeningeal disease (LMD) that has progressed after stereotactic radiosurgery (SRS) or whole brain radiation (WBRT). Presented at the American Society of Clinical Oncology Breast Cancer Symposium; San Francisco, CA. October 8-10, 2009; abstract \#2237

38. Roche H, Yelle L, Cognetti F, et al. Phase II clinical trial of ixabepilone (BMS-247550), an epothilone B analog, as first-line therapy in patients with metastatic breast cancer previously treated with anthracycline chemotherapy. J Clin Oncol. 2007; 25:3415-20. [PubMed: 17606972]

39. Schmid P, Kiewe P, Possinger K, et al. Phase I study of the novel, fully synthetic epothilone sagopilone (ZK-EPO) in patients with solid tumors. Ann Oncol. 2009

40. Thomas E, Tabernero J, Fornier M, et al. Phase II clinical trial of ixabepilone (BMS-247550), an epothilone B analog, in patients with taxane-resistant metastatic breast cancer. J Clin Oncol. 2007; 25:3399-406. [PubMed: 17606975]

41. Hoffmann J, Fichtner I, Lemm M, et al. Sagopilone crosses the blood-brain barrier in vivo to inhibit brain tumor growth and metastases. Neuro Oncol. 2009; 11:158-66. [PubMed: 18780814]

42. Hoffmann J, Vitale I, Buchmann B, et al. Improved cellular pharmacokinetics and pharmacodynamics underlie the wide anticancer activity of sagopilone. Cancer Res. 2008; 68:5301-8. [PubMed: 18593931]

43. Silvani A, Gaviani P, Fiumani A, et al. Systemic sagopilone (ZK-EPO) treatment of patients with recurrent malignant gliomas. J Neurooncol. 2009; 95:61-4. [PubMed: 19381446]

44. Peereboom DM, Supko JG, Carson KA, et al. A phase I/II trial and pharmacokinetic study of ixabepilone in adult patients with recurrent high-grade gliomas. J Neurooncol. 2010

45. Freedman RA, Bullitt E, Sun L, et al. A Phase II Study of Sagopilone (ZK 219477; ZK-EPO) in Patients With Breast Cancer and Brain Metastases. Clin Breast Cancer. 2011

46. Morrow P, Divers S, Provencher L, et al. Phase II study of sagopilone (ZK-Epo) in patients with recurrent metastatic breast cancer (MBC). J Clin Oncol. 2009; 27:15s. suppl; abstr 1083.

47. Bullitt E, Ewend MG, Aylward S, et al. Abnormal vessel tortuosity as a marker of treatment response of malignant gliomas: preliminary report. Technol Cancer Res Treat. 2004; 3:577-84. [PubMed: 15560715] 
48. Bullitt E, Gerig G, Pizer SM, et al. Measuring tortuosity of the intracerebral vasculature from MRA images. IEEE Trans Med Imaging. 2003; 22:1163-71. [PubMed: 12956271]

49. Murphy C, Nulsen B, Rump M, et al. Phase II trial of patupilone in patients with breast cancer brain metastases progressing or recurring after whole brain radiotherapy. Presented as abstract at 2009 Breast Cancer Symposium. abstract \#234.

50. Thomas FC, Taskar K, Rudraraju V, et al. Uptake of ANG1005, a novel paclitaxel derivative, through the blood-brain barrier into brain and experimental brain metastases of breast cancer. Pharm Res. 2009; 26:2486-94. [PubMed: 19774344]

51. Sarantopoulos, J.; Gabrail, NY.; Moulder, SL., et al. ANG1005: Results of a phase I study in patients with advanced solid tumors and brain metastases. Presented at the American Society of Clinical Oncology Annual Meeting; Chicago, IL. June 4-8, 2010; abstract \#2556

52. Drappatz, J.; Brenner, AJ.; Rosenfeld, S., et al. ANG1005: Results of a phase I study in patients with recurrent malignant glioma. J Clin Oncol; Presented at the American Society of Clinical Oncology Annual Meeting; Chicago, IL. June 4-8, 2010; 2010. abstract \#2009

53. Lin NU, Dieras V, Paul D, et al. Multicenter phase II study of lapatinib in patients with brain metastases from HER2-positive breast cancer. Clin Cancer Res. 2009; 15:1452-1459. In the largest multicenter protocol to date for women with breast cancer and progressive brain metastases, this report illustrates the clinical benefit of both single-agent lapatinib and lapatinib/ capecitabine for women with HER2-positive disease. [PubMed: 19228746]

54. Lin NU, Eierman W, Greil R, et al. Randomized phase II study of lapatinib plus capecitabine or lapatinib plus topotecan for patients with HER2-positive breast cancer brain metastases. $\mathrm{J}$ Neurooncol. 2011

55•. Bachelot TD, Romieu G, Campone M, et al. LANDSCAPE: An FNCLCC phase II study with lapatinib (L) and capecitabine (C) in patients with brain metastases (BM) from HER2-positive (+) metastatic breast cancer (MBC) before whole-brain radiotherapy (WBR). J Clin Oncol. 29:2011. (suppl; abstr 509). This report illustrates the clinical benefit of lapatinib/capecitabine in the upfront treatment of HER2-positive breast cancer brain metastases prior to receipt of WBRT. [PubMed: 21502544]

56. Lin NU, Ramakrishna N, Younger WJ, et al. Phase I study of lapatinib (L) in combination with whole-brain radiation therapy (WBRT) in patients (pts) with brain metastases from HER2-positive breast cancer. J Clin Oncol. 2010; 28:15s. suppl; abstr 1154.

57. De Azambuja E, Lemort M, Rossari JR, et al. Phase I study of lapatinib (L) and temozolomide (T) combination for the treatment of progressive brain metastases (BM) in HER2-positive metastatic breast cancer patients (Pts) (LAPTEM, LAP 111172). J Clin Oncol. 2011; 29 suppl; abstr 570.

58. Bullitt E, Lin NU, Smith JK, et al. Blood vessel morphologic changes depicted with MR angiography during treatment of brain metastases: a feasibility study. Radiology. 2007; 245:82430. [PubMed: 17954616]

59. Friedman HS, Prados MD, Wen PY, et al. Bevacizumab alone and in combination with irinotecan in recurrent glioblastoma. J Clin Oncol. 2009; 27:4733-40. [PubMed: 19720927]

60. Miller K, Wang M, Gralow J, et al. Paclitaxel plus bevacizumab versus paclitaxel alone for metastatic breast cancer. N Engl J Med. 2007; 357:2666-76. [PubMed: 18160686]

61. Besse B, Lasserre SF, Compton P, et al. Bevacizumab safety in patients with central nervous system metastases. Clin Cancer Res. 2010; 16:269-78. [PubMed: 20028762]

62. Donawho CK, Luo Y, Penning TD, et al. ABT-888, an orally active poly(ADP-ribose) polymerase inhibitor that potentiates DNA-damaging agents in preclinical tumor models. Clin Cancer Res. 2007; 13:2728-37. [PubMed: 17473206]

63. Anders, CK.; Nanda, R.; Liu, MC., et al. TBCRC 018: Phase II study of iniparib plus chemotherapy to treat triple-negative breast cancer (TNBC) brain metastases (BM). Presented at the American Society of Clinical Oncology Annual Meeting; Chicago, IL. June 3-7, 2011; abstract \#TPS127

64. Anders, CK.; Adamo, B.; Deal, AM., et al. Phosphatidylinositol 3-Kinase (PI3K) Pathway Activation in Breast Cancer Brain Metastases. Presented at the San Antonio Breast Cancer Symposium; San Antonio, TX. December 8-12, 2010; abstract \#P1-14-01 
65. World Health Organization. WHO Offset Publication No 48. Geneva: WHO; 1979. WHO Handbook for Reporting Results of Cancer Treatment.

66. Therasse P, Arbuck SG, Eisenhauer EA, et al. New guidelines to evaluate the response to treatment in solid tumors. European Organization for Research and Treatment of Cancer, National Cancer Institute of the United States, National Cancer Institute of Canada. J Natl Cancer Inst. 2000; 92:205-16. [PubMed: 10655437]

67. Kaiser K, Rauscher GH, Jacobs EA, et al. The import of trust in regular providers to trust in cancer physicians among white, African American, and Hispanic breast cancer patients. J Gen Intern Med. 2011; 26:51-7. [PubMed: 20811783]

68. Miller AB, Hoogstraten B, Staquet M, Winkler A. Reporting results of cancer treatment. Cancer. 1981; 47:207-14. [PubMed: 7459811]

69. Lin NU, Carey LA, Liu MC, et al. Phase II trial of lapatinib for brain metastases in patients with human epidermal growth factor receptor 2-positive breast cancer. J Clin Oncol. 2008; 26:1993-9. [PubMed: 18421051] 







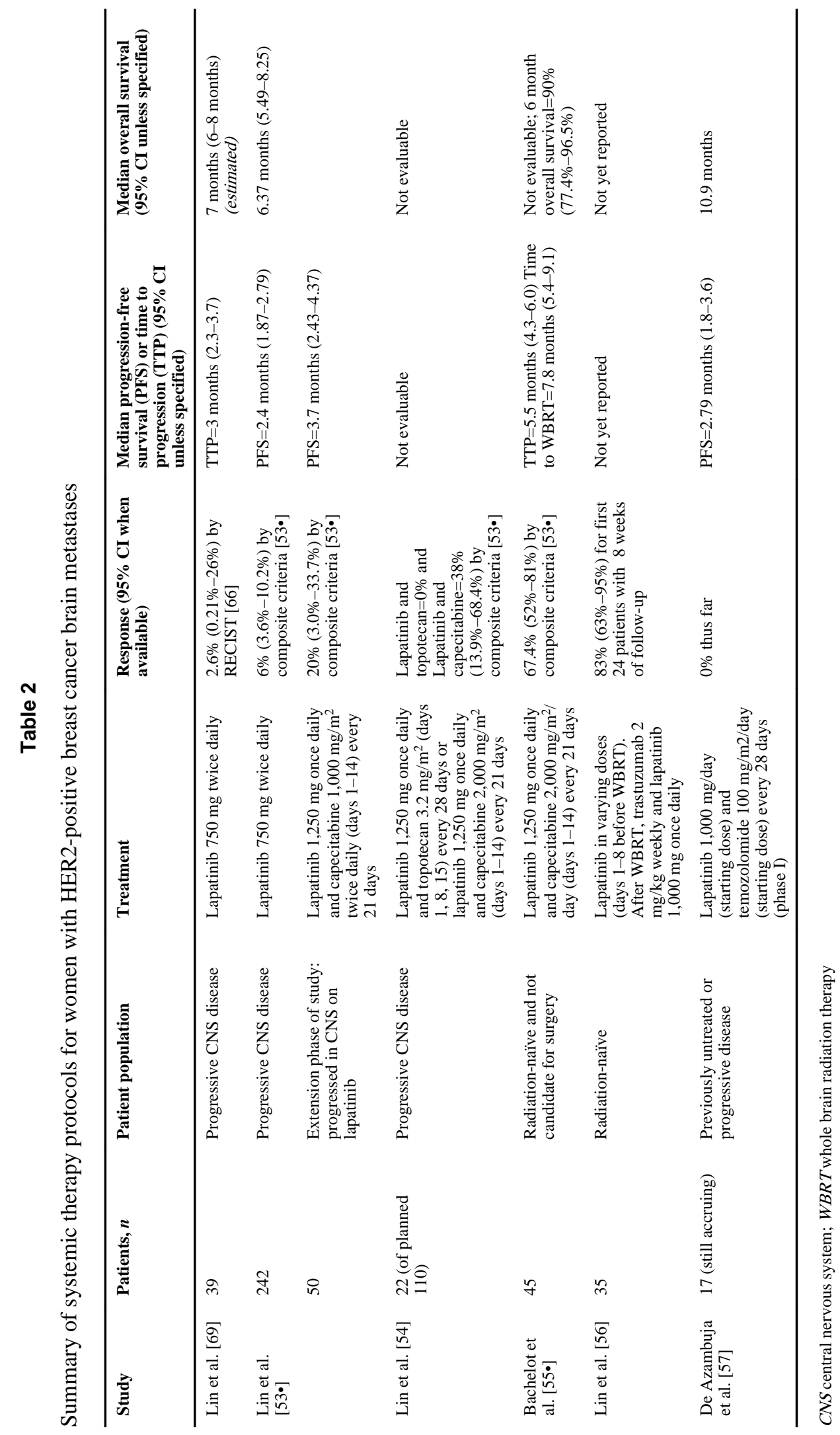

